\title{
Non-Mean-Field Behavior of the Contact Process on Scale-Free Networks
}

\author{
Claudio Castellano ${ }^{1}$ and Romualdo Pastor-Satorras ${ }^{2}$ \\ ${ }^{1}$ CNR-INFM, SMC, Dipartimento di Fisica, Università di Roma "La Sapienza", Piazzale Aldo Moro 2, I-00185 Roma, Italy \\ and CNR, Istituto dei Sistemi Complessi, Roma, Italy \\ ${ }^{2}$ Departament de Física i Enginyeria Nuclear, Universitat Politècnica de Catalunya, Campus Nord B4, 08034 Barcelona, Spain
} (Received 23 June 2005; published 24 January 2006)

\begin{abstract}
We present an analysis of the classical contact process on scale-free networks. A mean-field study, both for finite and infinite network sizes, yields an absorbing-state phase transition at a finite critical value of the control parameter, characterized by a set of exponents depending on the network structure. Since finite size effects are large and the infinite network limit cannot be reached in practice, a numerical study of the transition requires the application of finite size scaling theory. Contrary to other critical phenomena studied previously, the contact process in scale-free networks exhibits a nontrivial critical behavior that cannot be quantitatively accounted for by mean-field theory.
\end{abstract}

Recent years have witnessed an upsurge in the study of the structure and function of complex networks [1]. The interest in these systems has been driven by two motivations. First, a large amount of work has been devoted to the structural characterization of real networks, the main contribution being the discovery of their seemingly ubiquitous scale-free (SF) nature [2], characterized by a probability $P(k)$ that an element in the network (a vertex) is connected to other $k$ elements (has degree $k$ ), scaling as a power-law, $P(k) \sim k^{-\gamma}$, with a certain degree exponent $\gamma$ usually in the range $2<\gamma<3$ [2]. On the other hand, a great deal of attention has been drawn by the profound effects that a heterogeneous SF connectivity pattern can have on dynamical processes on complex networks. In particular, models exhibiting critical phase transitions such as ferromagnets [3], percolation [4], or epidemic models [5] have been studied on this class of networks. Present understanding of critical behavior in complex networks is essentially based on the application of mean-field (MF) techniques [6]. In the study of critical phenomena on regular substrates (lattices), MF theory plays a prominent role. Despite its simplicity, it describes qualitatively most phase transitions. Moreover, while in low spatial dimensions fluctuations cannot be neglected, above the upper critical dimension MF is even quantitatively correct, providing exact expressions for critical exponents. In the case of complex networks, since they usually fulfill the so-called small-world property [7], such systems can be viewed as infinite dimensional, and therefore one can expect MF to provide an exact description of dynamical processes taking place on networks, once degree heterogeneity has been taken into account. Results obtained so far seem to validate this idea, particularly for SF networks [8].

In this Letter we show the inadequacy of MF theory to describe some dynamical processes on SF networks, by considering the absorbing-state phase transition of the contact process (CP) [9]. We develop a MF theory for the $\mathrm{CP}$ on generic networks, and solve it for SF networks, obtaining expressions for both infinite and finite networks.
These expressions are compared with numerical simulations of the CP on SF networks, which are analyzed applying a finite size scaling (FSS) theory [10]. Our results show that the critical exponents of CP on SF networks cannot be accounted for by MF theory, and signal the presence of nontrivial fluctuation effects.

We consider the classical CP on generic networks, defined as follows [10]: An initial fraction $\rho_{0}$ of vertices is randomly chosen and occupied by a particle. The time evolution of the process runs as follows: At each time step $t$, a particle in a vertex $v_{i}$ is chosen at random. With probability $p$, this particle disappears. With probability $1-p$, on the other hand, the particle may generate an offspring. To do so, a vertex $v_{j}$, nearest neighbor of the vertex $v_{i}$, is randomly chosen. If vertex $v_{j}$ is occupied by a particle, nothing happens; if it is empty, a new particle is created on $v_{j}$. In any case, time is updated as $t \rightarrow$ $t+1 / n(t)$, where $n(t)$ is the number of particles present at the beginning of the time step [10]. In Euclidean $d$-dimensional lattices, the $\mathrm{CP}$ undergoes a continuous transition at a critical point $p_{c}$, separating an absorbing phase from an active one [10]. This phase transition is characterized in terms of the order parameter $\rho$, defined as the average density of particles in the steady state. For $p>p_{c}$, an absorbing phase with $\rho=0$ is observed. For $p<p_{c}$ the system sets in an active phase with a nonzero order parameter, obeying $\rho \sim \Delta^{\beta}$ for $p \rightarrow p_{c}^{-}$, where $\Delta=$ $p_{c}-p$. Close to $p_{c}$, the system is further characterized by diverging correlation length and time scales, namely $\xi \sim$ $|\Delta|^{-\nu_{\perp}}$ and $\tau \sim|\Delta|^{-\nu_{\|}}$. The critical exponents $\beta, \nu_{\perp}$, and $\nu_{\|}$provide a full characterization of the phase transition of $\mathrm{CP}$ in Euclidean lattices. Below the critical dimension $d_{c}=4$, the exponents are nontrivial, and depend explicitly on $d$. For $d>d_{c}$, the exponents take the classical MF values $\beta=\nu_{\|}=1, \nu_{\perp}=1 / 2$.

In order to gain analytical insight into the behavior of the $\mathrm{CP}$ on complex networks, we consider as usual a MF rate equation for the partial densities $\rho_{k}(t)$ of occupied vertices 
of degree $k$ [5], from which the total density is obtained as $\rho(t)=\sum_{k} \rho_{k}(t) P(k)$. The rate equation for the partial densities in a network characterized by a degree distribution $P(k)$ and degree correlations given by the conditional probability $P\left(k^{\prime} \mid k\right)$ that a vertex of degree $k$ is connected to a vertex of degree $k^{\prime}$ [11] can be thus written as [12]

$$
\partial_{t} \rho_{k}(t)=-\rho_{k}(t)+\lambda k\left[1-\rho_{k}(t)\right] \sum_{k^{\prime}} \frac{P\left(k^{\prime} \mid k\right) \rho_{k^{\prime}}(t)}{k^{\prime}} .
$$

The destruction term, due to the vanishing of particleswith probability $p$-at vertices of degree $k$, is proportional to the density of occupied vertices of degree $k, \rho_{k}(t)$. The creation term, on the other hand, is due to the generation of offspring from particles located at nearest neighbor vertices of empty vertices of degree $k$. Thus, it is proportional to the probability $\left[1-\rho_{k}(t)\right]$ that a vertex of degree $k$ is empty, that it is connected to a vertex of degree $k^{\prime}$, $P\left(k^{\prime} \mid k\right)$, that this one is occupied, $\rho_{k^{\prime}}(t)$, that it generates an offspring, $1-p$, and that this neighbor chooses to create the offspring on the vertex of degree $k$ under consideration, $\left(1 / k^{\prime}\right)$. In Eq. (1) we have performed a rescaling of time, introducing the new parameter $\lambda=(1-p) / p$. For uncorrelated networks, where $P\left(k^{\prime} \mid k\right)=k^{\prime} P\left(k^{\prime}\right) /\langle k\rangle$ [11], the rate equations take the form

$$
\partial_{t} \rho_{k}(t)=-\rho_{k}(t)+\lambda \frac{k}{\langle k\rangle}\left[1-\rho_{k}(t)\right] \rho(t) .
$$

The solution of Eq. (2) depends on the nature of the degree distribution. For homogeneous networks, in which $P(k)$ decays exponentially for large degrees, all vertices are approximately equivalent. Therefore, we have $k \simeq\langle k\rangle$ and $\rho_{k}(t) \simeq \rho(t)$, so that the rate equation reads $\partial_{t} \rho(t)=$ $-\lambda \rho^{2}(t)+\tilde{\Delta} \rho(t)$, where $\tilde{\Delta}=\lambda-1$. This equation describes the behavior of the $\mathrm{CP}$ in an infinite dimensional system; the critical point $\lambda_{c}=1\left(p_{c}=1 / 2\right)$ and the exponent $\beta=1$ are thus immediately found [10]. Moreover, from the solution $\rho(t) \sim \tilde{\Delta} / \lambda-e^{-\tilde{\Delta} t}$, we can identify the relevant characteristic time $\tau \sim \tilde{\Delta}^{-1}$ and the corresponding exponent $\nu_{\|}=1$.

For heterogeneous networks, with a degree distribution exhibiting large fluctuations, the solution of Eq. (2) must consider explicitly the $k$ dependence of the partial densities. Information on the active phase is obtained by imposing the steady state condition, $\partial_{t} \rho_{k}(t)=0$, which yields the nonzero solutions

$$
\rho_{k}=\frac{\lambda k \rho /\langle k\rangle}{1+\lambda k \rho /\langle k\rangle},
$$

where $\rho_{k}$ is now independent of time. In order to determine the critical behavior of the average density $\rho$ as a function of the control parameter $\lambda$ we focus on the self-consistent equation for the order parameter $\rho$. By combining Eq. (3) with the expression for $\rho$, one obtains

$$
\rho=\frac{\lambda \rho}{\langle k\rangle} \sum_{k} \frac{k P(k)}{1+\lambda k \rho /\langle k\rangle} .
$$

This equation depends on the full degree distribution. In the case of SF networks, for which the degree distribution in the continuous degree approximation is given by $P(k)=$ $(\gamma-1) m^{\gamma-1} k^{-\gamma}$, with $m$ the minimum degree in the network, the solution will depend on the degree exponent $\gamma$. Substituting the summation by an integral in Eq. (4), we obtain in the infinite network size limit (i.e., when the degree belongs to the range $[m, \infty])$ the expression $\rho=$ $F\left[1, \gamma-1, \gamma,-(\lambda \rho m /\langle k\rangle)^{-1}\right]$, where $F[a, b, c, z]$ is the Gauss hyper-geometric function [13]. To evaluate the critical behavior for small $\rho$, we invert this expression using the asymptotic expansion of the hyper-geometric function for $z \rightarrow-\infty$, obtaining [14] $\rho(\lambda) \sim(\lambda-1)^{1 /(\gamma-2)}$ for $2<$ $\gamma<3$ and $\rho(\lambda) \sim \lambda-1$ for $\gamma>3$. Hence the MF solution in infinite networks gives the critical exponent $\beta^{\mathrm{MF}}=$ $1 /(\gamma-2)$ for $\gamma<3$, and $\beta^{\mathrm{MF}}=1$ for $\gamma>3$, while the critical threshold is $\lambda_{c}^{\mathrm{MF}}=1\left(p_{c}^{\mathrm{MF}}=1 / 2\right)$, independent of the degree exponent.

The previous results, corresponding to networks of infinite size, are, however, strongly affected by finite size effects. The difference between an infinite SF network and one of finite size $N$ is that the latter has a cutoff or maximum degree $k_{c}$, which is a function of the network size [15], and that, for uncorrelated networks, scales as $k_{c}(N) \sim N^{1 / 2}$ [16]. The presence of the cutoff restricts the possible values of the degree to the range $\left[m, k_{c}\right]$. For finite networks, Eq. (4) yields, in the continuous degree approximation, $\rho=F\left[1, \gamma-1, \gamma,-(\lambda \rho m /\langle k\rangle)^{-1}\right]-$ $\left(k_{c} / m\right)^{1-\gamma} F\left[1, \gamma-1, \gamma,-\left(\lambda \rho k_{c} /\langle k\rangle\right)^{-1}\right]$. To obtain the MF critical behavior for a finite network, we evaluate this expression in the limit $\rho \rightarrow 0$ for generic $\rho k_{c}$, obtaining

$$
\rho=\frac{\langle k\rangle}{\lambda k_{c}} f\left(\frac{\langle k\rangle \tilde{\Delta} k_{c}^{\gamma-2}}{\lambda m^{\gamma-1}}\right),
$$

where $f(x)$ is the inverse of the function $g(x)=$ $\left(F[1, \gamma-1, \gamma,-1 / x]+\Gamma(\gamma) \Gamma(2-\gamma) x^{\gamma-1}\right) / x$. For $x \gg$ $1, f(x)$ diverges as $x^{1 /(\gamma-2)}$, in agreement with the infinite size limit $\rho \sim \tilde{\Delta}^{\beta^{\mathrm{MF}}}$. For small arguments, the function $f(x)$ vanishes for $x \rightarrow x_{0}^{+}$with $x_{0}>0$. Therefore, for finite $N$, if $\Delta$ is made smaller than a value proportional to $k_{c}^{2-\gamma}$, then $\rho=0$. This indicates that the effective critical point is shifted in the active phase in finite networks and that the convergence to the infinite network limit is slow, since corrections vanish as $N^{(2-\gamma) / 2}$, i.e., with an exponent smaller than 1.

From Eq. (5) we can ascertain when, in a system of $N$ vertices, finite size effects start to play a relevant role. It is clear that for $x<1$, i.e., $\tilde{\Delta}<\tilde{\Delta}_{\times} \sim\left(k_{c} / m\right)^{2-\gamma}$ such effects start to appear, so that one expects deviations from the decay $\rho \sim \tilde{\Delta}^{\beta^{\mathrm{MF}}}$. In terms of the order parameter this 


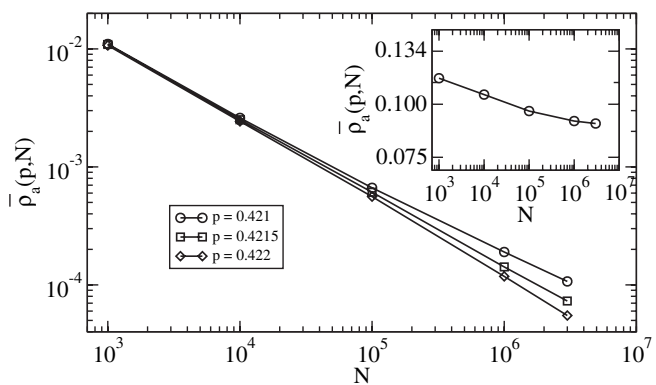

FIG. 1. $\bar{\rho}_{a}(p, N)$ as a function of the network size $N$ for the CP on UCM networks with $\gamma=2.75$, for values of $p$ close to $p_{c}$. Solid lines are guides to the eye. Inset: $\bar{\rho}_{a}(p, N)$ for $p=0.36$, showing that the infinite $N$ limit is not reached even far from $p_{c}$.

implies that finite size effects are negligible only if $\rho \gg$ $\rho_{\times}=\langle k\rangle /\left(\lambda k_{c}\right)$. This condition introduces serious constraints on the precision of the numerical estimate of the critical point when performed by measuring the dependence of the $\rho$ on $p$ in the active phase.

This difficulty can be overcome by using the FSS technique $[10,17]$. The FSS technique is based on the observation that, even below the critical point, the density of active sites in surviving runs $\bar{\rho}_{a}$ reaches a quasisteady state whose average is a decreasing function of the network size $N$ [10]. The FSS ansatz assumes the dependence of $\bar{\rho}_{a}$ as a function of $\Delta=p_{c}-p$ and $N$

$$
\bar{\rho}_{a}(\Delta, N)=N^{-\beta / \nu_{\perp}} f\left(\Delta N^{1 / \nu_{\perp}}\right),
$$

where the scaling function has the asymptotic behavior $f(x) \rightarrow x^{\beta}$ for $x \rightarrow \infty$, and $f(x) \rightarrow$ const for $x \rightarrow 0$. Compatibility with the theoretical behavior given in Eq. (5) for $x \gg 1$ requires that the exponents assume the MF values $\beta=1 /(\gamma-2)$ and $\nu_{\perp}=2 /(\gamma-2)$ in uncorrelated networks with cutoff scaling as $N^{1 / 2}$. Thus, at $p_{c}$ the function $\bar{\rho}_{a}$ is expected to decay as

$$
\bar{\rho}_{a}(0, N) \sim N^{-\beta / \nu_{\perp}} .
$$

This fact can be used to estimate the critical point, as the value of $p$ yielding a power-law behavior for $\bar{\rho}_{a}$ as a function of $N$. MF theory predicts in this case an exponent $\beta / \nu_{\perp}=1 / 2$.

In order to check these results, we have simulated numerically the $\mathrm{CP}$ on the uncorrelated configuration model (UCM) [18], which generates uncorrelated SF networks with arbitrary degree exponent and cutoff $k_{c}(N) \sim N^{1 / 2}$. We consider the particular cases $\gamma=2.75,2.5$, and 2.25. Simulations were performed with at least 100 runs of the $\mathrm{CP}$ for each realization of the network and no less than 100 such realizations for each value of $\gamma$ and $N$.

We focus first on the determination of the critical point and of the exponent ratio $\beta / \nu_{\perp}$ via Eq. (7). In Fig. 1 we display the density of active sites in surviving runs $\bar{\rho}_{a}$ as a function of the network size, for $\gamma=2.75$ and several values of the control parameter $p$. The critical point is

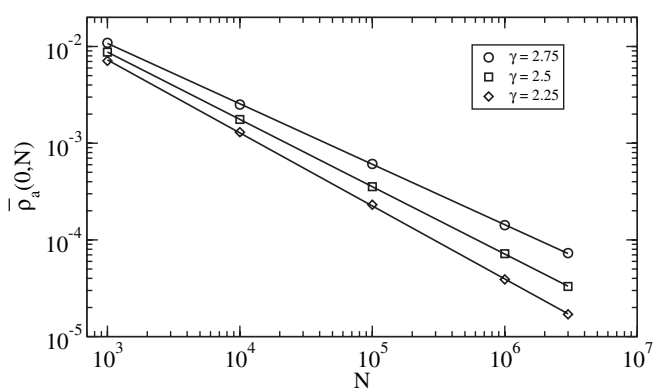

FIG. 2. Scaling of $\bar{\rho}_{a}(0, N)$ as a function of the network size for the CP on UCM networks for three values of $\gamma$. The critical points and exponents $\beta / \nu_{\perp}$ obtained are reported in Table I. Solid lines are least squares fits.

identified as the value of $p$ such that the decay of $\bar{\rho}_{a}$ vs $N$ is a pure power law, which for this particular value of $\gamma$ corresponds to $p_{c}=0.4215$. In Fig. 2 we show the decay of $\rho_{a}$ vs $N$ at criticality, for the three values of $\gamma$ considered. In all cases a very clear power-law behavior is identified, yielding the exponent ratios $\beta / \nu_{\perp}=0.63$ for $\gamma=2.75,0.70$ for $\gamma=2.50$, and 0.76 for $\gamma=2.25$. Notice that these values are in strong disagreement with the MF prediction $\beta / \nu_{\perp}=1 / 2$.

In principle the exponent $\beta$ should be determined by the way the steady state density of particles vanishes as a function of $p_{c}-p$ in the limit $N \rightarrow \infty$. However, it turns out that, even far from $p_{c}$, it is numerically impossible to fulfill the condition $\rho \gg \rho_{\times}$that guarantees the absence of finite size effects. This is apparent in the inset of Fig. 1, where one can see that even for $p=0.36$ the value of $\bar{\rho}_{a}$ slightly depends on $N$ even for large sizes. This very slow convergence hampers the determination of $\beta$ in the usual way.

The numerical value of $\beta$ and $\nu_{\perp}$ can, however, be determined via the FSS analysis, by noticing that from Eq. (6) we expect data for the surviving density to collapse onto a universal function $f(x)$ when plotting $N^{\beta / \nu_{\perp}} \bar{\rho}_{a}(\Delta, N)$ as a function of $\Delta N^{1 / \nu_{\perp}}$. Using the values

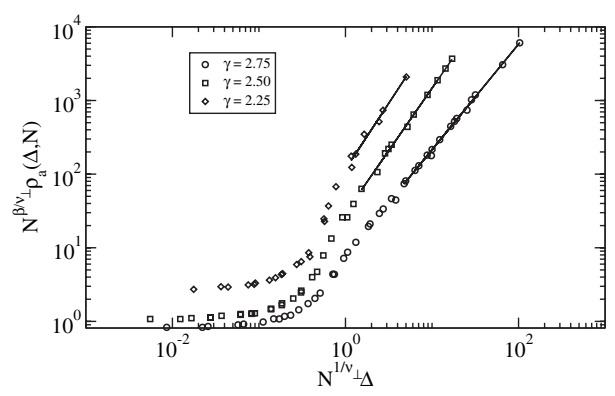

FIG. 3. Data collapse of $N^{\beta / \nu_{\perp}} \bar{\rho}_{a}(\Delta, N)$ as a function of $\Delta N^{1 / \nu_{\perp}}$ for the CP on UCM networks. Data are for system size ranging from $N=10^{3}$ to $N=10^{7}$. For clarity the data for $\gamma=2.25$ have been shifted upwards. The values of $\nu_{\perp}$ are those giving the best data collapses. The straight lines are least squares fits to the behavior $f(x) \rightarrow x^{\beta^{\prime}}$, for large $x$. 
TABLE I. Critical points and critical exponents for the CP on UCM networks, compared with MF values. Numbers in parentheses indicate the uncertainty in the last digit.

\begin{tabular}{ccccc}
\hline \hline & $p_{c}$ & $\beta / \nu_{\perp}$ & \multicolumn{1}{c}{$\beta$} & $\nu_{\perp}$ \\
\hline MF & $1 / 2$ & $1 / 2$ & $1 /(\gamma-2)$ & $2 /(\gamma-2)$ \\
$\gamma=2.75$ & $0.4215(5)$ & $0.63(4)$ & $1.52(5)$ & $2.4(2)$ \\
$\gamma=2.50$ & $0.4425(5)$ & $0.70(3)$ & $2.0(1)$ & $2.8(3)$ \\
$\gamma=2.25$ & $0.465(3)$ & $0.76(5)$ & $2.4(1)$ & $3.2(3)$ \\
\hline \hline
\end{tabular}

of $\beta / \nu_{\perp}$ obtained from Fig. 1 we try the collapse for various values of $\beta$ (and, as a consequence, of $\nu_{\perp}$ ). The best data collapses, shown in Fig. 3, are obtained for the values of $\beta$ and $\nu_{\perp}$ reported in Table I. The scaling function $f(x)$ is expected to diverge as $x^{\beta}$ for asymptotically large $x$. We measure how the numerical scaling functions go for large $x$ in Fig. 2, finding the effective behavior $f(x) \sim x^{\beta^{\prime}}$ with $\beta^{\prime}=1.43,1.70$, and 1.79. The disagreement with the values of $\beta$ providing the best data collapse indicates that the values of $x$ that can be reached are not large enough to see the truly asymptotic $x^{\beta}$ behavior.

We summarize the results obtained in Table I. The conclusion of this analysis is that the CP in SF networks exhibits an absorbing-state phase transition at a nonzero critical point, whose exponents are not correctly predicted by MF theory [19]. This result is surprising: it is generally believed that dynamical models on SF networks find in this formalism a correct description, once degree heterogeneity has been taken into account [5]. The results presented here open two clear perspectives for future work. The first is the quest for a theory beyond $\mathrm{MF}$ for the $\mathrm{CP}$ on heterogeneous networks. The second is the investigation of the limits of MF theory for generic dynamical processes on heterogeneous topologies. A useful indication along these lines is provided by Fig. 4, where we plot the ratio of the standard deviation $\Delta \rho_{k}$ over the mean density $\rho_{k}$ restricted to vertices of degree $k$, at the critical point. It turns out that the fluctuations are much larger than the average except for the smallest $k$, all the more so for increasing system size. Hence it is incorrect to assume (as MF theory does) that the

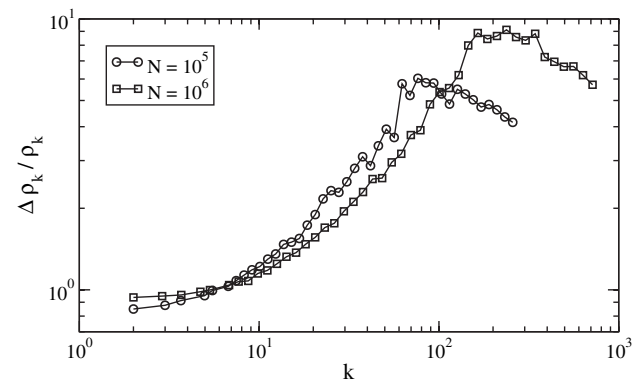

FIG. 4. Ratio of the standard deviation $\Delta \rho_{k}$ of the distribution of the density of occupied vertices of degree $k$ over the average $\rho_{k}$, versus the degree $k$, for $\gamma=2.75, p=p_{c}=0.4215$ and two values of the system size $N$. mean $\rho_{k}$ provide all information about the distribution of densities for vertices of a certain degree. The inclusion of these large fluctuations and the understanding of their role are thus key points to develop a theory beyond MF, able to fully capture the physics of collective phenomena on SF networks.

We thank Alessandro Vespignani for useful discussions. R.P.-S. acknowledges financial support from the Spanish MEC (FEDER), under Project No. FIS2004-05923-C02-01 and additional support from the MCyT and DURSI (Spain). C.C. acknowledges financial support from Azione Integrata Italia-Spagna IT1792/2004.

[1] R. Albert and A.-L. Barabási, Rev. Mod. Phys. 74, 47 (2002); S. N. Dorogovtsev and J. F. F. Mendes, Evolution of Networks: From Biological Nets to the Internet and $W W W$ (Oxford University Press, Oxford, 2003).

[2] A.-L. Barabási and R. Albert, Science 286, 509 (1999).

[3] M. Leone et al., Eur. Phys. J. B 28, 191 (2002); S. N. Dorogovtsev, A. V. Goltsev, and J.F.F. Mendes, Phys. Rev. E 66, 016104 (2002).

[4] R. Cohen et al., Phys. Rev. Lett. 85, 4626 (2000); D. S. Callaway et al., Phys. Rev. Lett. 85, 5468 (2000).

[5] R. Pastor-Satorras and A. Vespignani, Phys. Rev. Lett. 86, 3200 (2001); Y. Moreno, R. Pastor-Satorras, and A. Vespignani, Eur. Phys. J. B 26, 521 (2002); R. PastorSatorras and A. Vespignani, Phys. Rev. E 63, 066117 (2001).

[6] H.E. Stanley, Introduction to Phase Transitions and Critical Phenomena (Oxford University Press, Oxford, 1971).

[7] D. J. Watts and S. H. Strogatz, Nature (London) 393, 440 (1998).

[8] A. V. Goltsev, S. N. Dorogovtsev, and J.F. F. Mendes, Phys. Rev. E 67, 026123 (2003).

[9] T. E. Harris, Ann. Probab. 2, 969 (1974).

[10] J. Marro and R. Dickman, Nonequilibrium Phase Transitions in Lattice Models (Cambridge University Press, Cambridge, 1999).

[11] M. Boguñá, R. Pastor-Satorras, and A. Vespignani, Lect. Notes Phys. 625, 127 (2003).

[12] M. Catanzaro, M. Boguñá, and R. Pastor-Satorras, Phys. Rev. E 71, 056104 (2005).

[13] M. Abramowitz and I. A. Stegun, Handbook of Mathematical Functions (Dover, New York, 1972).

[14] The case $\gamma=3$ gives logarithmic corrections that will be discussed elsewhere.

[15] S. N. Dorogovtsev and J.F. F. Mendes, Adv. Phys. 51, 1079 (2002).

[16] M. Boguñá, R. Pastor-Satorras, and A. Vespignani, Eur. Phys. J. B 38, 205 (2004).

[17] D. H. Zanette, Phys. Rev. E 64, 050901 (2001); P. R. A. Campos, V. M. de Oliveira, and F. G. Brady Moreira, Phys. Rev. E 67, 026104 (2003).

[18] M. Catanzaro, M. Boguñá, and R. Pastor-Satorras, Phys. Rev. E 71, 027103 (2005).

[19] We have checked that for complete and random graphs MF predictions are instead verified. 